\title{
Suivi hydrologique par altimétrie spatiale dans le bassin du Congo
}

\author{
Christophe Brachet $^{1}$, Alice Andral ${ }^{2}$, Georges Gulemvuga Guzanga ${ }^{3}$, Blaise Léandre Tondo ${ }^{3}$, \\ Pierre-Olivier Malaterre ${ }^{4}$, and Sébastien Legrand ${ }^{5}$ \\ ${ }^{1}$ OiEau, Paris, France \\ ${ }^{2} \mathrm{CNES}$, Toulouse, France \\ ${ }^{3}$ CICOS, Kinshasa, RD Congo \\ ${ }^{4}$ G-EAU, Univ Montpellier, AgroParisTech, BRGM, CIRAD, IRD, INRAE, Institut Agro, Montpellier, France \\ ${ }^{5} \mathrm{CNR}$, Lyon, France \\ Correspondence: Christophe Brachet (c.brachet@oieau.fr) \\ Published: 16 November 2021
}

\begin{abstract}
Résumé. Le satellite SWOT sera lancé fin 2022 par le Centre National d'Etudes Spatiales (CNES) français et la National Aeronautics and Space Administration (NASA) américaine. L'altimétrie spatiale permet de compléter les données hydrométriques in situ à travers l'établissement de «stations virtuelles », au croisement de la trace au sol du satellite avec un cours d'eau. SWOT améliorera encore la couverture des zones observées ainsi que la précision grâce à une technologie innovante. Un projet d'appui à la Commission Internationale du bassin Congo-Oubangui-Sangha (CICOS) développé depuis 2016 sur financement de l'Agence Française de Développement (AFD) et facilité par l'Office International de l'Eau (OiEau) permet de promouvoir l'hydrologie spatiale à travers un groupe d'institutions françaises, en appui à la CICOS. Diverses activités ont été développées dont la fourniture d'une base de données spatiales par l'Institut de Recherche pour le Développement (IRD), ainsi que la comparaison avec des données in situ. Une méthodologie innovante a été proposée par l'Institut national de recherche pour l'agriculture, l'alimentation et l'environnement (INRAE) pour passer des altitudes aux débits aux stations virtuelles. Des applications spécifiques ont par ailleurs été développées par la Compagnie Nationale du Rhône (CNR) pour l'hydroélectricité et la navigation fluviale.
\end{abstract}

\section{Introduction}

Depuis plusieurs décennies, on constate un fort déclin des réseaux hydrométriques dans le monde et en particulier en Afrique. Le Congo, plus grand fleuve d'Afrique, n'échappe pas à ce constat. Mais plusieurs innovations ou évolutions permettent de surmonter ces manques : l'OMM a par exemple développé HydroHub (Dixon et al., 2020) ou encore WHOS (WMO Hydrological Observing System - Pecora et Lins, 2020), et des approches multidisciplinaires sont promues (Tauro et al., 2018). L'altimétrie spatiale, qui consiste à mesurer les hauteurs d'eau par satellite est une de ces innovations majeures.

Sept entités françaises se sont ainsi regroupées au sein d'un groupe de travail pour valoriser l'altimétrie spatiale dans le bassin du Congo et proposer des applications opérationnelles.

Après avoir rappelé le contexte, le présent article présente le projet d'appui du groupe de travail sur le bassin du Congo ainsi que les principaux résultats obtenus : augmentation de la base de données de stations virtuelles sur le Congo, étude comparative des données spatiales et in situ au droit d'un même site, transformation des altitudes en débits et utilisation pour des applications opérationnelles (navigation fluviale et hydroélectricité). 


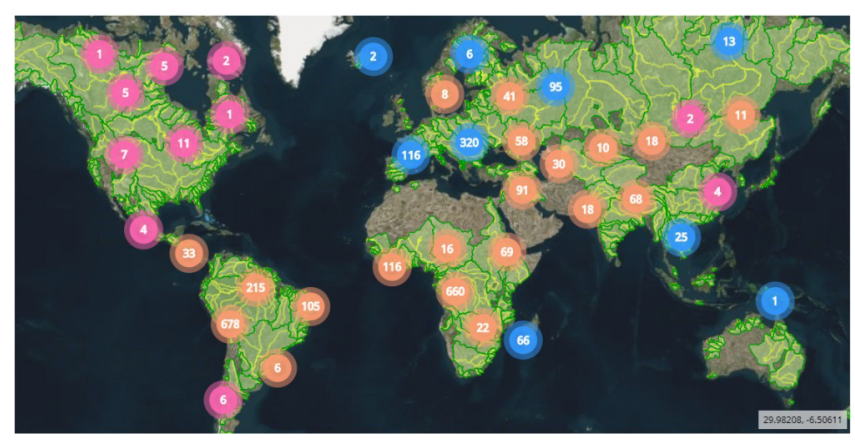

Figure 1. Stations virtuelles disponibles sur Hydroweb pour le suivi des lacs et rivières.

\section{Contexte}

\subsection{L'altimétrie spatiale}

L'altimétrie spatiale repose sur les satellites équipés d'un appareil radar (altimètre). Les mesures sont réalisées au droit de «stations virtuelles », qui correspondent aux intersections de la trajectoire du satellite et des cours d'eau.

Les mesures de hauteur d'eau par altimétrie sont accessibles sur le site Hydroweb (http://hydroweb.theia-land.fr, la date du dernier accès : 22 octobre 2021). Hydroweb est une base de données de variations des niveaux d'eau sur les lacs et les fleuves du monde à partir de l'altimétrie satellitaire, qui a été progressivement mise en place depuis 2003 (Crétaux et al., 2011). Aujourd'hui, ce sont plus de 11000 stations virtuelles qui sont disponibles partout dans le monde (Fig. 1).

L'altimétrie spatiale permet ainsi de compléter le réseau de mesure in situ afin de densifier les données et d'assurer un meilleur suivi hydrologique (Calmant et Seyler, 2006; Calmant et al., 2008).

\subsection{Le groupe de travail sur l'hydrologie spatiale}

Le Groupe de travail sur l'hydrologie spatiale a été créé en 2014 puis consolidé par un accord signé lors de la COP22 à Marrakech. Il regroupe les institutions françaises suivantes : le CNES, l'IRD, l'AFD, l'OiEau (animateur du groupe), l'INRAE, BRLi et CNR.

Les travaux du groupe s'intéressent à la valorisation des données existantes d'altimétrie spatiale (en particulier avec les missions Jason et Sentinel3A) pour des applications opérationnelles sur des bassins versants, et à la préparation des applications aval pour le futur satellite SWOT. Il permet également de capitaliser les acquis et avancées obtenues.

\subsection{La CICOS et le bassin du Congo}

Le bassin du Congo est le second bassin au monde après celui de l'Amazone avec une superficie de $3,7 \times 10^{6} \mathrm{~km}^{2}$ et un débit moyen de $41000 \mathrm{~m}^{3} \mathrm{~s}^{-1}$.
La CICOS est une organisation intergouvernementale regroupant l'Angola, le Cameroun, la Centrafrique, le Congo, la République Démocratique du Congo et le Gabon. Elle a été créée en 1999 à travers l'Accord créant la CICOS et instituant un Régime Fluvial Uniforme (CICOS, 1999), complété en 2007 (CICOS, 2007) par un mandat de Gestion Intégrée des Ressources en Eau du bassin du Congo. En décembre 2015 lors de la COP 21 à Paris, une convention a été signée entre la CICOS et l'AFD afin de renforcer le suivi hydrologique dans le bassin du Congo en tenant compte de l'adaptation aux changements climatiques.

\section{Le projet de suivi hydrologique du bassin du Congo}

\subsection{Objectifs et activités du projet}

Le groupe de travail a choisi le bassin du Congo comme bassin pilote avec les objectifs spécifiques suivants : (1) l'établissement d'une base de données de stations virtuelles, (2) l'étude de la conversion des élévations altimétriques en débits, (3) l'élaboration d'un système d'information hydrologique et (4) les applications sur l'hydroélectricité et la navigation.

\subsection{Etablissement d'une base de données de stations virtuelles}

Le projet a permis de mettre en œuvre près de 550 stations virtuelles sur l'ensemble du bassin du Congo (CICOS, 2016 b, c). La calibration d'une station virtuelle a été réalisée avec une station in situ (Maluku-Trechot) installée au droit d'une trace de satellite. La précision estimée de la mesure altimétrique est ainsi de $14 \mathrm{~cm}$ (Fig. 2). Cette précision sera encore accrue avec SWOT, dont les incertitudes seront moindres.

\subsection{Passage des altitudes aux débits}

Le passage des altitudes aux débits est l'un des chantiers importants en cours au sein du groupe. Diverses méthodologies ont été développées ces dernières années dans le cadre du projet SWOT et du DAWG (Discharge Algorithm Working Group) pour estimer les débits des fleuves à partir des futures données SWOT et de manière plus générale, à partir de données issues des mesures satellitaires altimétriques et de masques d'eau (Durand et al., 2016; Gejadze et Malaterre, 2016, 2017; Oubanas et al., 2018a, b).

La méthodologie décrite dans ces articles Oubanas et al. (2018a, b) et dénommée SIC4DVar fonctionne avec des données de type purement SWOT (hauteur, largeur, pente), et est donc adaptée à un contexte non-jaugé sans aucune donnée in situ complémentaire. Si des données complémentaires sont disponibles, la méthode peut les intégrer (bathymétrie, frottements, autres données satellitaires, etc.) 


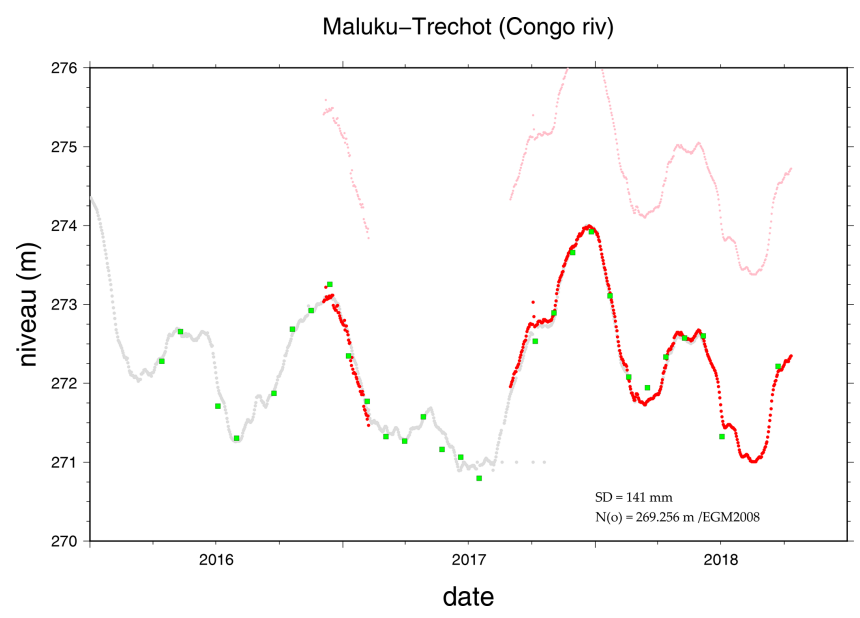

Figure 2. Comparaison entre les hauteurs d'eau estimées par altimétrie avec l'altimètre Sentinel 3 et les stations de Maluku-Trechot et de Brazzaville situées de part et d'autre de la trace altimétrique.

Des étapes de cette méthodologie ont été appliquées au fleuve Congo et sur l'Oubangui pour estimer les débits à partir des données altimétriques disponibles depuis 2002 grâce à diverses missions : Envisat, Saral, Jason 2 et 3, Sentinel 3A. Les données sont utilisées dans un algorithme d'inversion de l'équation de Manning-Strickler pour calculer une bathymétrie, ainsi que pour positionner et dimensionner les chutes qui sont détectées automatiquement.

Le modèle hydraulique généré avec la bathymétrie et les frottements obtenus permet ensuite différentes exploitations en simulation. Une exploitation du modèle est de générer des courbes de tarage $Q(Z)$ par des séries de calculs en régime permanent pour différents débits couvrant les gammes réalistes observées sur le terrain. Une fois ces courbes de tarage générées, elles peuvent être exploitées de manière opérationnelle et en temps réel pour calculer des débits à partir des cotes altimétriques satellitaires.

Même si de nombreuses étapes sont perfectibles et en cours de tests, les résultats obtenus et validés sur des données in situ sont très encourageants, par exemple sur l'Oubangui avec une très bonne reconstitution de la $Q(Z)$ in situ (RootMean-Square Error RMSE $=3 \%$ ), sur le Congo avec une très bonne reconstitution de la bathymétrie dans les zones où des relevés $\mathrm{ADCP}$ étaient disponibles.

\subsection{Applications pour l'hydroélectricité et la navigation sur le bassin du Congo}

\subsubsection{Navigation}

La Sangha, affluent de rive droite transfrontalier du fleuve Congo, draine un bassin versant de $213000 \mathrm{~km}^{2}$. Le transport fluvial est un enjeu majeur sur cette rivière, qui n'est pas navigable toute l'année. La hauteur d'eau lue quotidiennement à l'échelle limnimétrique d'Ouesso par le GIE-
SCEVN (Groupement d'Intérêt Économique - Service Commun d'Entretien des Voies Navigables pour les deux États Congo et Centrafrique), est utilisée comme indicateur de navigabilité sur le fleuve Sangha.

Un modèle opérationnel de prévision des hauteurs d'eau à Ouesso au pas de temps journalier a ainsi été développé afin d'anticiper les arrêts de navigation ou d'adapter le chargement des bateaux. Ce modèle statistique, calé sur des données allant jusqu'à 2012, utilise les données de hauteur d'eau à Ouesso, ainsi que les données de précipitations GPCP (Adler et al., 2018).

L'ajout des données d'altimétrie spatiale dans le modèle a été étudié. L'apport des données a été mesuré par l'évolution du $R^{2}$ ajusté du modèle avec et sans les stations virtuelles sur la même période. Les résultats sont contrastés, mais d'autres types de modélisation (comme la modélisation distribuée avec assimilation des données spatiales), pourraient s'avérer plus adaptées aux données spatiales.

\subsubsection{Hydroélectricité}

L'hydroélectricité est par ailleurs un des enjeux identifiés dans le cadre du SDAGE de la CICOS (Schéma Directeur d'Aménagement et de Gestion des Eaux, CICOS, 2016a), dans un contexte où les populations locales ont un accès limité à l'électricité.

CNR a déterminé les tronçons les plus propices au développement de l'hydroélectricité dans les bassins du Congo et de l'Ogooué. La méthodologie a été établie à partir d'un indicateur de puissance hydroélectrique, calculée comme le produit du débit et de la pente du cours d'eau. Le débit a été reconstitué par similitude des bassins versants, à partir des débits des stations in situ et des données de précipitations du Climatic Research Unit (University of East Anglia). La pente entre deux stations consécutives est calculée comme la différence entre l'altitude moyenne aux stations virtuelles.

Des tronçons avec un potentiel hydroélectrique intéressant ont ainsi été identifiés, et correspondent à des zones de fort débit et/ou de forte pente. A l'inverse, des zones à plus faible potentiel sont apparues, telles que la Cuvette Centrale. La confrontation de ces résultats avec les projets hydroélectriques déjà identifiés sur le bassin confirme l'intérêt de la méthodologie comme outil d'aide à la décision permettant à la CICOS d'identifier les tronçons les plus intéressants et d'étudier ultérieurement des sites à équiper, en coopération avec les Etats. Néanmoins certaines limitations sont apparues, comme le manque de données dans les zones de montagne ou sur les cours d'eau de moindre largeur. L'utilisation d'un MNT suffisamment précis pourrait constituer une alternative à l'utilisation de l'altimétrie.

\section{Le programme SWOT : présentation et attentes}

Le programme satellitaire SWOT (Surface Water and Ocean Topography) du CNES et de la NASA est un pro- 

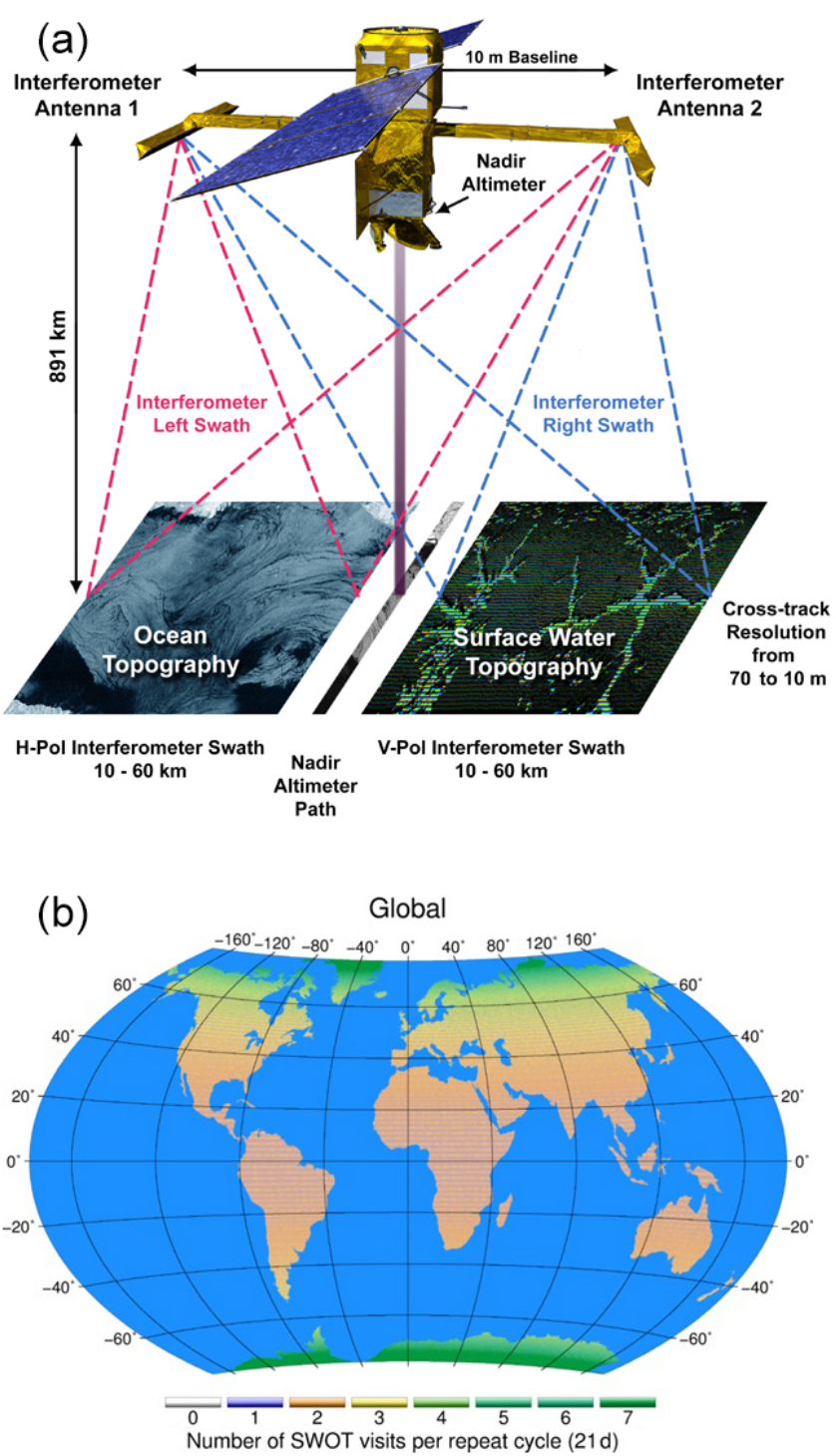

Figure 3. (a) Principes de la mesure SWOT (en haut). (b) Couverture spatiale et temporelle de SWOT (nombre de revisites de SWOT par cycle de 21 jours) (en bas).

jet de satellite d'observation de la Terre qui fournira à l'horizon 2023 et pour la première fois les variations spatiotemporelles des niveaux d'eau à l'échelle globale des grands fleuves, lacs et rivières et les niveaux des océans. Par rapport aux missions précédentes, SWOT propose une nouvelle technologie non encore testée depuis l'espace, l'altimétrie à large fauchée (Biancamaria et al., 2016, cf. Fig. 3).

L'altimétrie à large fauchée permettra d'obtenir des données continues, sur des tronçons de cours d'eau d'au moins $100 \mathrm{~m}$ de large, et non plus des données ponctuelles. Il est prévu que les données soient mises à disposition par biefs d'environ $10 \mathrm{~km}$ de long, ainsi qu'à des résolutions plus fines (ex. : sections tous les $200 \mathrm{~m}$ et même pixels de quelques dizaines de $\mathrm{m}^{2}$ ). La précision attendue de SWOT d'environ
$8 \mathrm{~cm}$ à l'échelle des biefs. La technologie à large fauchée permettra d'obtenir une couverture globale des altitudes, des plans d'eau et de pente, ce qui constituera un progrès par rapport à l'altimétrie conventionnelle.

\section{Conclusion}

L'altimétrie spatiale se présente comme une technologie innovante de mesure hydrologique au côté du réseau classique des stations in situ. La densification du réseau de mesure par les stations virtuelles sur le bassin du Congo, grâce aux différentes missions satellitaires altimétriques existantes, est un réel atout dans le suivi hydrologique de ce grand bassin transfrontalier et l'amélioration de la connaissance de ses ressources en eau.

Les appuis du groupe de travail sur l'hydrologie spatiale à la CICOS et l'arrivée prochaine du satellite SWOT et ses produits avals, permettront l'amélioration de la gestion et de la prise de décision quant à l'utilisation des ressources en eau du bassin du Congo (navigation fluviale, hydroélectricité, forêts inondées et biodiversité...) et d'autres grands bassins transfrontaliers (Niger et Sénégal en particulier), toujours en complémentarité des observations de terrain qui restent indispensables.

Disponibilité du code. Le code des logiciels utilisés n'est pas du domaine public. Il est la propriété de la CICOS qui représente ses 6 Etats membres.

Disponibilité des données. Les données utilisées ne sont pas du domaine public. Elles sont la propriété de la CICOS qui représente ses 6 Etats membres.

Collaborateurs. AA du Centre National d'Etudes Spatiales a mis en œuvre avec les collègues de l'Institut de Recherche pour le Développement les activités du projet relatives à l'altimétrie spatiale. POM de l'Institut national de recherche pour l'agriculture, l'alimentation et l'environnement a plus spécifiquement développé l'outil de Passage des altitudes aux débits. SL de la Compagnie Nationale du Rhône a réalisé les applications pour l'hydroélectricité et la navigation sur le bassin du Congo. GGG et BLT de la Commission Internationale du bassin Congo-Oubangui-Sangha ont contribué à l'ensemble des activités, en tant que maître d'ouvrage. CB de l'Office International de l'Eau a préparé et coordonné la rédaction de l'article avec la contribution de tous les co-auteurs.

Intérêts concurrents. Les auteurs déclarent qu'ils n'ont aucun conflit d'intérêts. 
Clause de non-responsabilité. Publisher's note : Copernicus Publications remains neutral with regard to jurisdictional claims in published maps and institutional affiliations.

Déclaration du numéro spécial. This article is part of the special issue "Hydrology of Large River Basins of Africa". It is a result of the 4th International Conference on the "Hydrology of the Great Rivers of Africa", Cotonou, Benin, 13-20 November 2021.

Remerciements. Nos vifs remerciements vont à Gil Mahé de l'Institut de Recherche pour le Développement.

Financement. This research has been supported by the Agence Française de Développement (grant no. CZZ2054).

\section{Références}

Adler, R., Wang, J., Sapiano, M., Huffman, G., Bolvin, D., Nelkin, E., and NOAA CDR Program : Global Precipitation Climatology Project Climate Data Record, Version 1.3 (Daily) Precipitation, NOAA National Centers for Environmental Information, https://doi.org/10.7289/V5RX998Z, 2018.

Biancamaria, S., Lettenmaier, D. P., and Pavelsky, T. M. : The SWOT mission and its capabilities for land hydrology, Surv. Geophys., 37, 307-337, https://doi.org/10.1007/s10712015-9346-y, 2016.

Calmant, S. and Seyler, F. : Continental surface waters from satellite altimetry, C. R. Geosci., 338, 1113-1122, https://doi.org/10.1016/j.crte.2006.05, 2006.

Calmant, S., Seyler, F., and Cretaux, J.-F. : Monitoring Continental Waters by Satellite Altimetry, Surv. Geophys., 29, 247-269, https://doi.org/10.1007/s10712-008-9051-1, 2008.

CICOS : Accord instituant un régime fluvial uniforme et créant la CICOS, accessible à l'adresse suivante : https: //www.cicos.int/wp-content/uploads/Accord-Instituant-un-R\% C3\%A9gime-Fluvial-Uniforme-et-cr\%C3\%A9ant-la-CICOS... ...-2.pdf (la date du dernier accès : 22 octobre 2021), 1999.

CICOS : Additif à l'Accord instituant un régime fluvial uniforme et créant la CICOS, accessible à l'adresse suivante : https://www. cicos.int/wp-content/uploads/Additif_a_1_Accord_Instituant_ un_Regime_Fluvial_Uniforme_et_creant_la_CICOS.pdf (la date du dernier accès : 22 octobre 2021), 2007.

CICOS : "Schéma Directeur d'Aménagement et de Gestion des Eaux de la CICOS. Programme de Mesures 2016-2020”, 161 pp., accessible à l'adresse suivante : https://www.cicos.int/ publications/cicos-sdage-programme-des-mesures/ (la date du dernier accès : 22 octobre 2021), 2016 a.

CICOS : Rapport sur l'amélioration et la validation de données altimétriques spatiales sur le bassin du Congo, CICOS, Kinshasa, RD Congo, 45 pp., 2016 b.
CICOS : Rapport sur la mise à disposition de données altimétriques satellitaires à la CICOS, CICOS, Kinshasa, RD Congo, 17 pp., 2016c.

Cretaux, J. F., Jelinski, W., Calmant, S., Kouraev, A., Vuglinski, V., Berge Nguyen, M., Gennero, M-C., Nino, F., Abarca Del Rio, R., Cazenave, A., and Maisongrande, P. : SOLS : A lake database to monitor in the Near Real Time water level and storage variations from remote sensing data, Adv. Space Res., 47, 14971507, https://doi.org/10.1016/j.asr.2011.01.004, 2011.

Dixon, H., Sandström, S., Cudennec C., Lins H., Abrate T., Bérod D., Chernov I., Ravalitera N., Sighomnou D., and Teichert F. : Intergovernmental cooperation for hydrometry - what, why, how ?, Hydrolog. Sci. J., Special Issue : Hydrological data : opportunities and barriers, https://doi.org/10.1080/02626667.2020.1764569, 2020.

Durand, M., Gleason, C., Garambois, P.-A., Bjerklie, D., Smith, L., Roux, H., Rodriguez, E., Bates, P., Pavelsky, T., and Monnier, J. : An intercomparison of remote sensing river discharge estimation algorithms from measurements of river height, width, and slope, Water Resour. Res., 52, 4527-4549, https://doi.org/10.1002/2015WR018434, 2016.

Gejadze, I. Y. and Malaterre, P.-O. : Design of the control set in the framework of variational data assimilation, J. Comput. Phys., 325, 358-379, https://doi.org/10.1016/j.jcp.2016.08.02, 2016.

Gejadze, I. Y. and Malaterre, P.-O. : Discharge estimation under uncertainty using variational methods with application to the full Saint-Venant hydraulic network model, Int. J. Numer. Meth. Fl., 83, 405-430, https://doi.org/10.1002/fld.4273, 2017.

Oubanas, H., Gejadze, I. Y., Malaterre, P.-O., and Mercier, F. : River discharge estimation from synthetic SWOTtype observations using variational data assimilation and the full Saint-Venant model, J. Hydrol., 559, 638-647, https://doi.org/10.1016/j.jhydrol.2018.02.004, 2018a.

Oubanas, H., Gejadze, I. Y., Malaterre, P.-O., Durand, M., Frasson, R., Wui, R., and Domeneghetti, A. : Discharge estimation in ungauged basins through variational data assimilation: The potential of the SWOT mission, Water Resour. Res., 54, 2405-2423, https://doi.org/10.1002/2017WR021735, 2018b.

Pecora, S. and Lins, H. : E-monitoring the nature of water, Hydrolog. Sci. J., 65, 683-698, https://doi.org/10.1080/02626667.2020.1724296, 2020.

Tauro, F., Selker, J., van de Giesen, N., Abrate, T., Uijlenhoet, R., Porfiri, M., Manfreda, S., Caylor, K., Moramarco, T., Benveniste, J., Ciraolo, G., Estes, L., Domeneghetti, A., Perks, M., Corbari, C., Rabiei, E., Ravazzani, G., Bogena, H., Harfouche, A., Brocca, L., Maltese, A., Wickert, A., Tarpanelli, A., Good, S., Lopez Alcala, J. M., Petroselli, A., Cudennec, C., Blume, T., Hut, R., and Grimaldi, S. : Measurements and observations in the XXI century : innovation and multi-disciplinarity to sense the hydrological cycle, Hydrolog. Sci. J., 63, 169-196, https://doi.org/10.1080/02626667.2017.1420191, 2018. 